\title{
THE EFFECT OF RUPTURE STARTING POINT UNCERTAINTY ON THE LONG PERIOD GROUND MOTION PREDICTION OF TOKAI EARTHQUAKE
}

\author{
Toshio MAEGAWA ${ }^{1)}$, Mitoshi YASUI ${ }^{2)}$ and Yoshiaki HISADA ${ }^{3)}$ \\ ${ }^{11}$ Member, Technical Research \& Development Institute, Kumagai Gumi Co., Ltd., M. Eng. \\ ${ }^{2)}$ Member, Research and Development Center, Toda Corporation, M. Eng. \\ ${ }^{3}$ Member, Professor, Kogakuin University, Dr. Eng.
}

\begin{abstract}
The effect of rupture starting point uncertainty on the prediction of long-period ground motion using a source model was examined regarding the hypothetical Tokai Earthquake. Monte Carlo simulation with a sample size of 30,000 was conducted assuming that the rupture starting point is uniformly distributed in the fault to calculate the statistics of the ground motion. The results of numerical simulations revealed that the maximum values of velocity response spectra in the long period region are well expressed by TYPE III extreme value distribution. The presence of an upper limit in this statistical distribution was also pointed out by using the extreme value statistics theory.
\end{abstract}

Key Words: Tokai Earthquake, Long Period Earthquake Ground Motion, Rupture Starting Point, Extreme Value Statistics Theory

\section{INTRODUCTION}

In recent years, earthquake ground motion has been calculated considering the seismic source process by using various computational techniques, such as the stochastic Green's function method, with various fault parameters, for instance, the fault plane size and earthquake moments. On the other hand, fault parameters involve significant uncertainty, with some parameters being difficult to assume even with the latest seismological knowledge. Dealing with the expected Tokai Earthquake, the authors examined the effect of uncertainty in the rupture starting point on the predicted long-period ground motion.

Midorikawa et al. ${ }^{1)}$ examined the statistics of characterized seismic fault models for 19 earthquakes in Japan, and reported that asperities tend to be located near the bottom end of the fault plane in strike-slip faultings, while being slightly fewer near both sides. Also, rupture starting points are reported to be located mostly in the lower half of an asperity. Sekiguchi et al. ${ }^{2)}$ examined the effect of dispersion in source parameters, such as slip velocity, stress drop value, and rupture starting point, in relation to strong ground motion prediction of the expected Nankai Earthquake. They pointed out that the calculated ground motion is greatly affected by the setting of the position of the rupture starting point, which alters the rupture directivity. Regarding ground motion evaluation obtained using fault models, Okano ${ }^{3)}$ comprehensively discussed dispersions that can be involved in such evaluation, 
Table 1 Fault parameters of the Tokai Earthquake

\begin{tabular}{|c|c|c|c|}
\hline & & This study & Hazard map \\
\hline \multicolumn{4}{|l|}{ DOuter fault parameters } \\
\hline Strike angle & (deg.) & 205,235 & Mean $220(205-235)$ \\
\hline Dip angle & (deg.) & 10,20 & Mean 15 (10-20) \\
\hline Rake angle & (deg.) & 90 & 90 \\
\hline Area & $S\left(\mathrm{~km}^{2}\right)$ & 9,500 & About 9,400 \\
\hline Stress drop & $\Delta \delta_{C}(\mathrm{MPa})$ & 3.0 & 3.0 \\
\hline Earthquake moment & $M_{O}\left(\mathrm{~N}^{*} \mathrm{~m}\right)$ & $1.09 \times 10^{21}$ & $\frac{1.12 \times 10^{21}}{\left(=S^{1.5} \Delta \delta_{c} /\left(7 \pi^{1.5} / 16\right)\right)}$ \\
\hline Moment magnitude & & 8.0 & 8.0 \\
\hline Rigidity & $\mu\left(\mathrm{N} / \mathrm{m}^{2}\right)$ & $3.50 \times 10^{10}$ & $3.48 \times 10^{10}$ \\
\hline Average dislocation & $D(\mathrm{~m})$ & 3.28 & 3.42 \\
\hline \multicolumn{4}{|l|}{ Inner fault parameters } \\
\hline \multicolumn{4}{|l|}{ Asperity } \\
\hline Area & $S_{a}\left(\mathrm{~km}^{2}\right)$ & 1,700 & About $1,786(=0.19 \mathrm{~S})$ \\
\hline Average dislocation & $D_{a}(\mathrm{~m})$ & 6.93 & $7.18(=2.1 \mathrm{D})$ \\
\hline Earthquake moment & $M_{o a}\left(\mathrm{~N}^{*} \mathrm{~m}\right)$ & $3.91 \times 10^{20}$ & $4.46 \times 10^{20}\left(=\mu S_{a} D_{a}\right)$ \\
\hline Stress drop & $\Delta \delta_{a}(\mathrm{Mpa})$ & 15.8 & $15.8\left(=\Delta \delta_{c} \times S / S_{a}\right)$ \\
\hline Number of aspeirties & & 3 & 3 \\
\hline \multicolumn{4}{|l|}{ Background } \\
\hline Area & $S_{b}\left(\mathrm{~km}^{2}\right)$ & 7,800 & About 7,614 \\
\hline average Dislocation & $D_{h}(\mathrm{~m})$ & 2.51 & 2.54 \\
\hline Earthquake Moment & $M_{o b}\left(\mathrm{~N}^{*} \mathrm{~m}\right)$ & $6.98 \times 10^{20}$ & $6.73 \times 10^{20}$ \\
\hline$f_{\max }$ & $\delta_{b}(\mathrm{~Hz})$ & 6 & 6 \\
\hline \multicolumn{4}{|l|}{ Other parameters } \\
\hline Rupture propergation mode & & Concentric-ring-like & Concentric-ring-like \\
\hline Rupture velocity & $V_{r}(\mathrm{~km} / \mathrm{s})$ & 2.7 & 2.7 \\
\hline
\end{tabular}

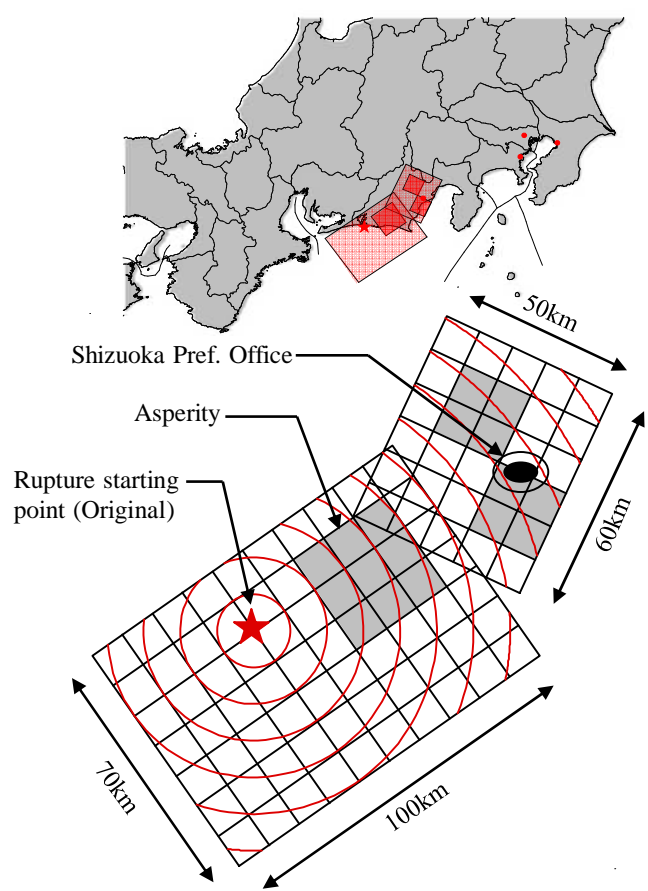

Fig. 1 Source region enlarged view

referring to a wide variety of related parameters. He argues that, when using the results of such evaluation for seismic design, it is necessary to consider the variability of indeterminate parameters and present the expected value and the variance (fluctuation band) to the extent conceivable. This paper reports on the results of numerical simulation in which the rupture starting point is assumed to be uniformly distributed in the fault plane in consideration of its uncertainty, while determinately giving such parameters as the number and position of the asperities and the stress drop value, with reference to past research results ${ }^{4), 5), 6)}$. As already mentioned, uncertainty of the rupture starting point has been dealt with in past studies, but such investigation has mostly been limited to only a few cases of parametric study, and is insufficient for considering ground motion of the maximum conceivable level. By performing Monte Carlo simulation (MCS) with a sufficiently large sample size, this study focuses on long-period ground motions with an exceedance probability of around $10^{-4}$.

Similarly to the rupture starting point, uncertainty of the asperity position may have an influence on the calculated ground motion ${ }^{7}$, but it is expected that the result has some degree of generality because of the assumption that the rupture starting point is uniformly distributed over the fault plane. That is, all possible cases are almost entirely covered by the tripartite positional relationship between the rupture starting point located in the entire area of the fault plane, the asperity which is placed near the fault plane center, and the calculation point of the ground motion. The past studies referred to in this study state that the predicted hypocentral region of the Tokai earthquake corresponds to the region unruptured by the Tonankai earthquake in 1944 (Showa Tonankai earthquake). The area of the rupture starting point of the expected Tokai earthquake may therefore be narrowed to a certain extent. However, it is of practical significance in seismic design to grasp the probability density of long-period ground motions calculated based on an assumption of the maximum possible variation range.

\section{SOURCE MODELING AND SYNTHESIS OF EARTHQUAKE MOTION}

Source modeling was carried out using the 2009 trial version of "Long Period Ground Motion Hazard Maps of Japan")" (hereafter referred to as the "Hazard Map") issued by the Headquarters for Earthquake Research Promotion as a reference. In this Hazard Map, three-dimensional underground 
structure models are used and the source region has a curved elliptical shape along the plate boundary, but in this study the fault is replaced with two simple rectangular planes assuming a layered soil model, and the source parameters were set so that both models would be as nearly equal as possible, considering the fault area of the three-dimensional model, the relationship between the total slip and the asperity slip and the average modulus of rigidity of the layered soil model. Regarding the asperity and background area, the differences between the both models of the earthquake moments were designed to be $10 \%$ or less. Table 1 compares the source parameters of this study with those of the Hazard Map. It should be noted that, as to the area where the two rectangular fault planes overlap, the slip value of the overlapped portion of the north fault is assumed to be 0 in this study. The approximation expression proposed by Nakamura and Miyatake ${ }^{8)}$ is adopted for the source time function. Two fault planes, the north and south, are set as shown in Fig. 1, and the sum of the ground motion calculated for each is evaluated. The deep subsurface structure was modeled as layered soil based on the data at each point of ground motion calculation on the Hazard Map.

As the synthesis method of earthquake motion, a theoretical approach based on the wave number integration method proposed by Hisada ${ }^{9}$ is adopted. The long period component of the ground motion greater than $3.5 \mathrm{sec}$ (long period ground motion) is focused on, while the surface layer (S-wave velocity less than $350 \mathrm{~m} / \mathrm{s}$ ) of each calculation point is ignored, because its effects on the response spectrum in this period region is insignificant.

\section{CALCULATION RESULTS OF LONG PERIOD GROUND MOTION}

\section{Comparison with the Hazard Map}

As described in the previous chapter, we adopted a simple model assuming a parallel-layered soil structure and rectangular fault shape, although the Hazard Map regarding the same Tokai earthquake was computed based on the finite difference method with a three-dimensional underground structure model. This simplified model has an advantage of shortening the computation time, because the calculation cost is enormous in MCS considering the uncertainty of the rupture starting point. This section compares these two models and examines the accuracy of the predicted results of the simplified model.

Figure 2 shows a comparison between long period ground motion calculations by the Hazard Map and this study. This study focuses on the maximum values of velocity response spectra in the long period region in consideration of the seismic design spectra for long period structures. Regarding 4 sites shown in Fig. 2 (a) (d), the prefectural capitals of Shizuoka, Kanagawa, Tokyo and Chiba, the results by both methods do not precisely agree in all directions. In the waveform of the Hazard Map for Tokyo and Chiba at 200 to $300 \mathrm{sec}$ in the horizontal directions, long-lasting and developing long-period ground motion is found, but no such waveform is found in our study results. This is presumably due to the fact that the simple parallel-layered model adopted in this study is incapable of expressing the effect of the characteristic deep soil structures of the Kanto Plain ${ }^{6), 10)}$, which is described well by the three-dimensional finite difference method used for the Hazard Map. For evaluating the duration time of the long-period ground motion in the Kanto Plain, the phases of subsequent waveform and input energy by the earthquake to the long period structures, there is an accuracy problem in this study method. Therefore, verification by high-precision models remains a challenge for the future. On the other hand, when focusing only on the maximum values of the response spectra in the period range of 3.5 to $20 \mathrm{sec}$, the simplified model of this study is capable of providing acceptable prediction values in comparison with the finite difference method of the Hazard Map, though the maximum periods do not agree with the latter. In consideration of the trade-off between accuracy and computational cost, the effect of the uncertainty of the rupture starting point on the predicted long-period motion is examined by this simple model in the following sections.

Effect of the position setting of the rupture starting point on the long-period ground motion The numerically calculated results of the long-period ground motions (EW direction components) at 
the Tokyo Metropolitan Government Office and Shizuoka Prefectural Office are shown in Fig. 3, with the rupture starting points being placed at the same location as the Hazard Map (hereafter referred to as the "Original"), as well as at the north edge of the north fault. The resulting long-period ground motion in the case where rupture starts from the north edge is considerably smaller than the case where rupture starts from the Original both in Tokyo and Shizuoka. Such a tendency is a fact well known to many researchers and structural seismic design engineers, and it is customary to conduct parametric calculation with multiple rupture starting points to avoid underestimation ${ }^{11), 12}$. When the long-period ground motion varies greatly depending on the location of the rupture starting point, such as at Tokyo in this example, accountability is desirable for justification regarding position setting. However, in the current knowledge of seismology, it is generally not always easy to precisely estimate the rupture starting point within a fault ${ }^{13)}$. In the following sections, the authors assume the rupture starting point location to be a random variable with uncertainty, and examine its effect by numerical experiments using the MCS technique.

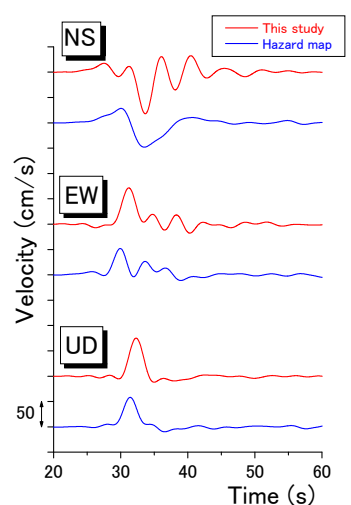

(a) Shizuoka Pref. Office

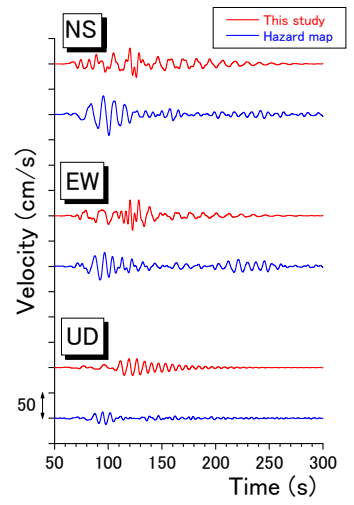

(c) Tokyo Metropolitan Government
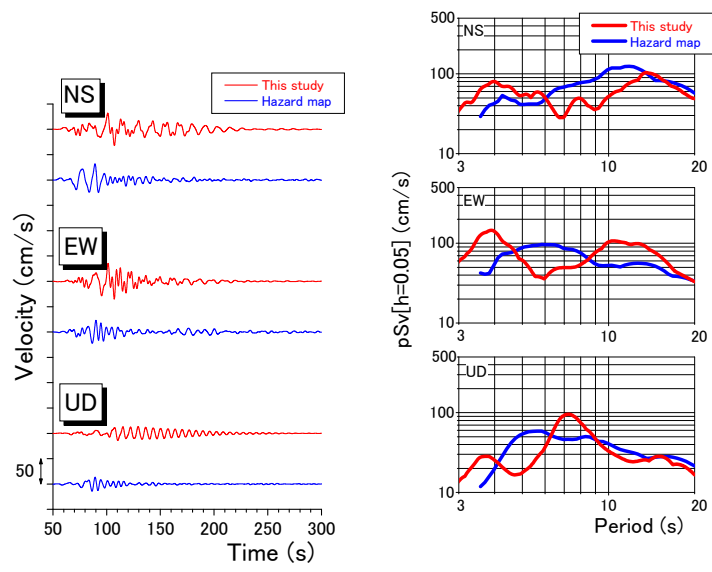

(b) Kanagawa Pref. Office
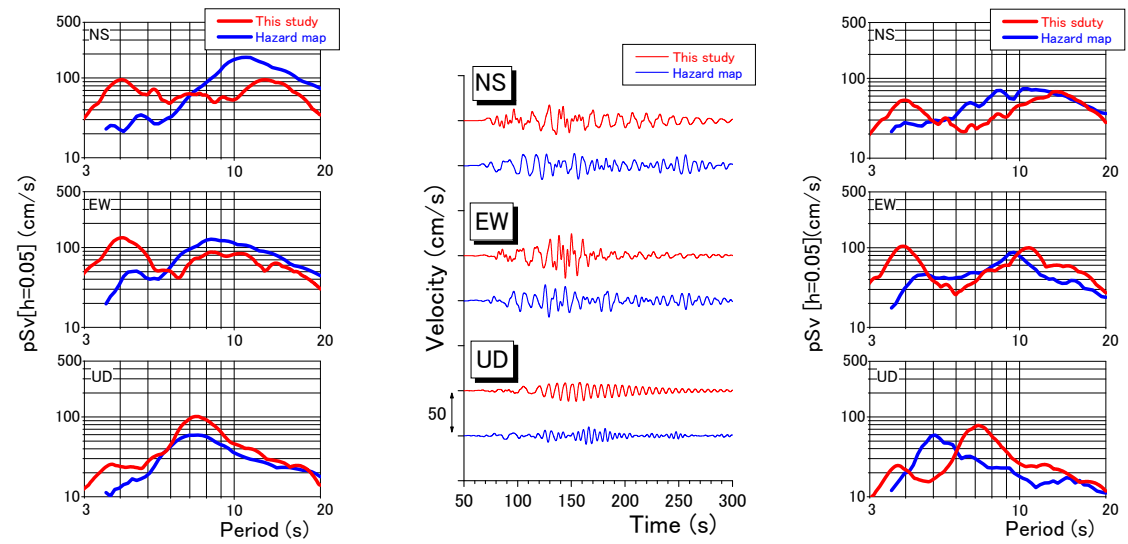

(d) Chiba Pref. Office

regarding long-period ground motion calculation

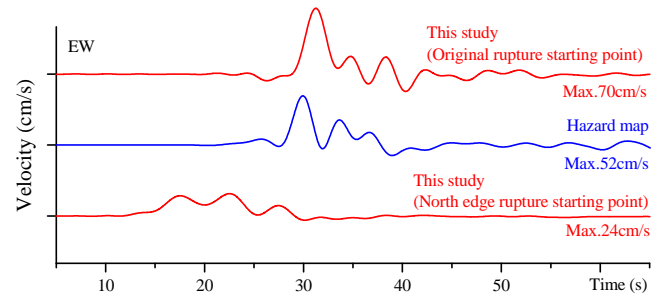

(a) Shizuoka Pref. Office

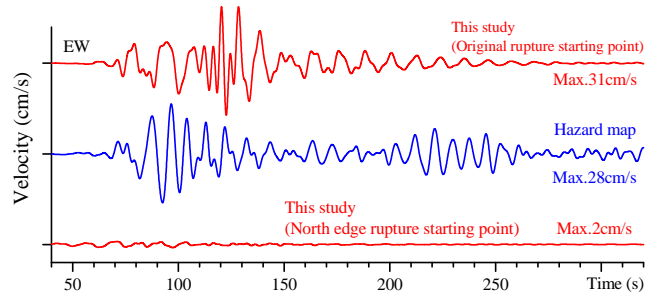

(b) Tokyo Metropolitan Government

Fig. 3 Long-period ground motion with rupture starting point at the north edge of the north fault 


\section{MCS with the rupture starting point in the south fault}

Figure 4 shows the statistics of the long-period ground motion velocity spectra at Shizuoka and Tokyo obtained by MCS on the assumption that the rupture starting point is uniformly distributed in the south fault and rupture propagates in a concentric manner. A sample size of 500 was adopted here, as only the expected response and coefficient of variation (C.O.V: $\sigma / \mu)$ are dealt with. It has been confirmed that a greater sample size scarcely changes the expected value and C.O.V. It has previously been reported that rupture starting points generally tend to exist outside of the asperity ${ }^{14), 15)}$, but here the rupture starting point is assumed to be uniformly distributed over the entire fault plane including the asperity area to allow simple interpretation of the results. Note that the effect of this assumption on the results is verified later in this paper by numerical calculation using a simpler source model. Figure 4 also shows the results with the Original rupture starting point. The response spectra at Tokyo nearly agree with the expected value plus one sigma. It can therefore be said that the Original setting of the rupture starting point provides an input ground motion for seismic design of structures to achieve a certain level of adequacy on the safe side, as far as comparison with MCS is concerned. The Hazard Map says that the rupture starting point was "set near the end of the Tokai slow event". It is also inferred that attention was paid to avoid underestimation of the ground motion of the populous urban areas, such as Tokyo ${ }^{16)}$. On the other hand, the results of Shizuoka EW with the Original are below the upper and mean levels of MCS, not leading to evaluation on the safe side.
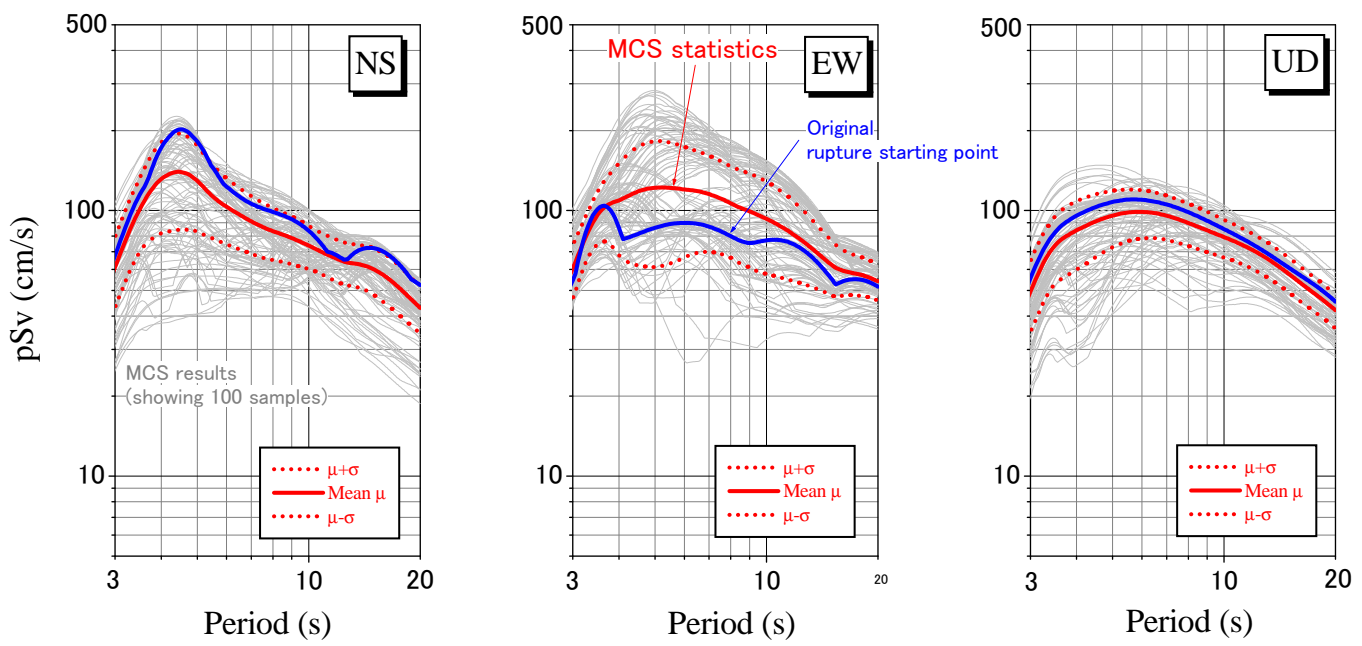

(a) Shizuoka Pref. Office
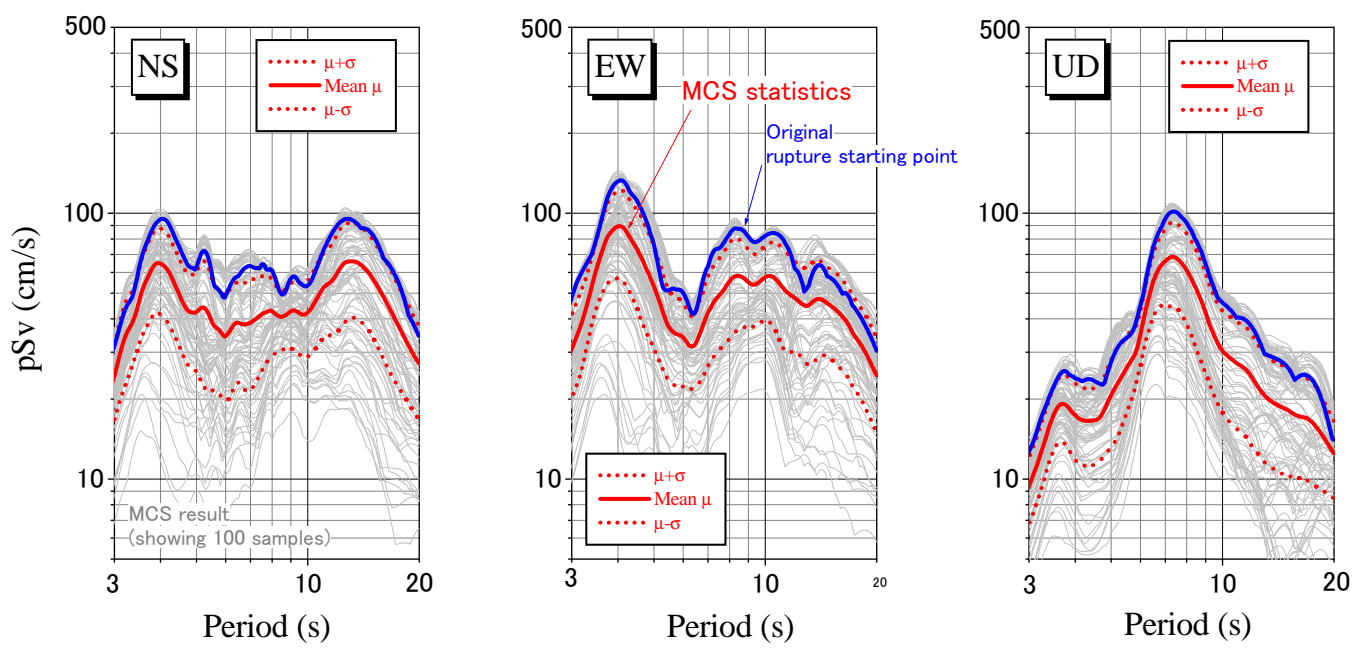

(b) Tokyo Metropolitan Government

Fig. 4 Statistics of Velocity Response Spectrum $(\mathrm{h}=0.05)$ by MCS 
MCS with two independent centers of rupture propagation in both north and south faults

In this section, MCS is conducted assuming two independent random points as centers of rupture propagation to evaluate the sum of the long period ground motion waveforms calculated for the north and south faults, incorporating the time difference obtained by dividing the center-to-center distance by the rupture propagation velocity $\operatorname{Vr}(=2.7 \mathrm{~km} / \mathrm{s})$ into the result of north fault calculation. In other words, rupture is assumed to occur from one point in the south fault, and its effect then propagates to the north fault at a velocity of Vr. When the rupture wavefront arrives at another point in the north fault, the north fault rupture starts around there. The reason for this setting of the centers of rupture propagation is that the positional relationship of the rupture center and the asperity can cause strong long-period ground motion at the point of calculation. Figure 5 shows the probability density functions of the maximum values of the velocity response spectra at the Tokyo Metropolitan Government Office and Shizuoka Prefectural Office determined by MCS. The sample size of MCS is 30,000, because statistical distribution is discussed. This figure also shows the lognormal distribution and TYPE-I extreme value distribution (Gumbel distribution, double exponential distribution) estimated by the method of moments, and TYPE-III extreme value distribution (Weibull distribution) calculated by the method of maximum likelihood. The reason for the estimation by extreme value distribution is that the target is the statistical distribution of the maximum values of the response spectra in the period region of 3.5 to $20 \mathrm{sec}$. The extreme value distribution is a distribution that the maximum and minimum values of $n$ samples extracted from the same population will follow ${ }^{17)}$. Here each value of velocity response spectra in the above-mentioned period region is regarded as a statistic contained in the same population, and a velocity response spectrum is regarded as the sample group. The extreme value distribution is thus used for estimation as a distribution that the maximum values of velocity response spectra follow.

The probability density function of Generalized Extreme Value ${ }^{18)}$ (GEV) distribution can be expressed by

$$
f_{X}(x)=\frac{1}{\sigma} \exp \left[-\left(1+k \frac{x-\mu}{\sigma}\right)^{-\frac{1}{k}}\right]\left(1+k \frac{x-\mu}{\sigma}\right)^{-1-\frac{1}{k}}
$$

where $k, \sigma$, and $\mu$ are a shape parameter, scale parameter and location parameter, respectively.

By putting

in Equation (1),

$$
K=-\frac{1}{k}, \quad \theta=-\frac{\sigma}{k}, \quad \mu^{\prime}=\mu+\theta
$$

$$
f_{X}(x)=\frac{K}{\theta} \exp \left[-\left(-\frac{x-\mu^{\prime}}{\theta}\right)^{K}\right]\left(-\frac{x-\mu^{\prime}}{\theta}\right)^{K-1}
$$

is derived, and this formula is consistent with TYPE-III extreme value distribution. When estimating the parameters in equation (1) by the maximum likelihood estimation from the sample values obtained by the MCS, if $\mathrm{k}$ is a negative value, GEV distribution gives TYPE-III extreme value distribution, and the factor in the equation (1)

$$
1+k \frac{x-\mu}{\sigma}
$$

must be a positive value. From this fact, the upper limit, $\operatorname{Max}[\mathrm{X}]$, in a statistical distribution of the maximum value, $\mathrm{X}$, is determined as follows: 


$$
\operatorname{Max}[X]=\mu-\frac{\sigma}{k}
$$

Figure 5 shows that the distribution that well represents the results of the MCS is TYPE-III extreme value distribution. Especially in the small value range of a hazard (the probability that the velocity response spectrum of long-period ground motion exceeds a specific value) of $10^{-2}$ to $10^{-4}$, TYPE-III gives more accurate results of distribution than the other two. Table 2 gives the upper limit values of the velocity response spectra estimated by GEV (Max[X]), the maximum values of the samples obtained by MCS, and $0.1 \%, 1 \%$, and $5 \%$ exceedance probability spectrum values (by MCS) in each direction. Although these values are greater than the values that are commonly used in the current seismic design practice of structures, it is not always necessary to apply the same design criteria to these strong ground motions. It is rather important to make effective use of the relationship between the ground motion strength and its exceedance probability for structural seismic design. As one example of such use, the seismic capacity of structural system safety may be confirmed with respect to long-period ground motion with a $1 \%$ exceedance probability in addition to normal seismic design. Such design criteria in proportion to exceedance probability should be established by taking into consideration the importance of the structure and the social influence of its function loss or destruction. For structures for which a very high level of safety is required, a hazard of $0.1 \%$ or less may be taken into account.

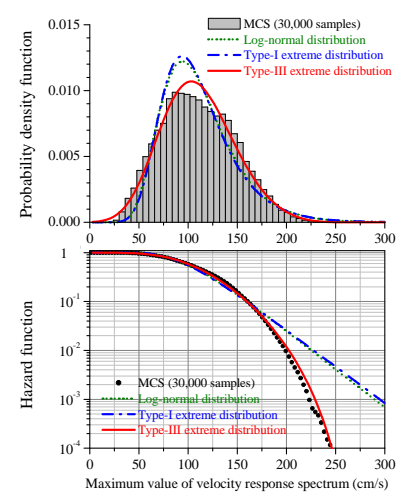

(a-1) NS direction

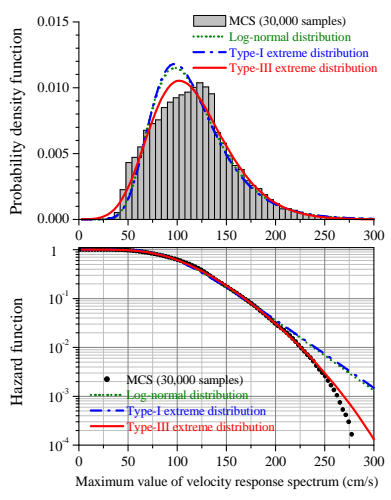

(a-2) EW direction (a) Shizuoka Pref. Office

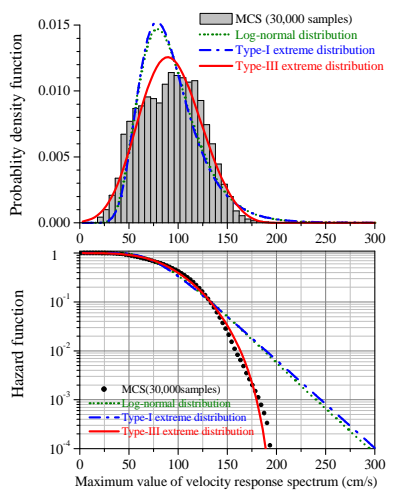

(a-3) UD direction

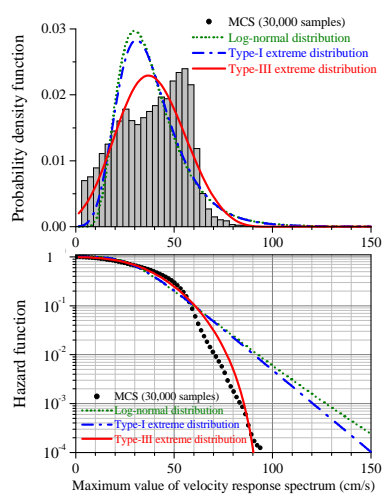

(b-1) NS direction

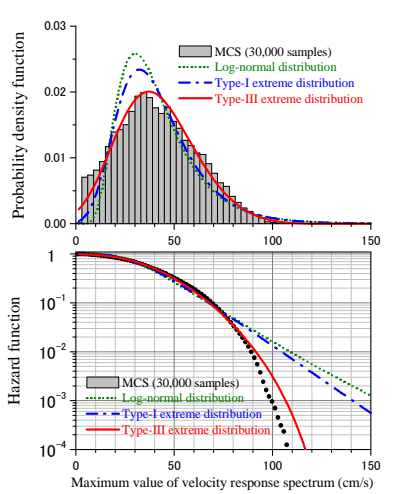

(b-2) EW direction

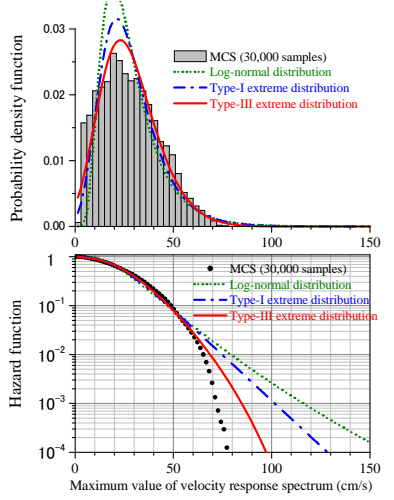

(b-3) UD direction

(b) Tokyo Metropolitan Government

Fig. 5 Hazard function and probability density of the maximum value of the velocity response spectrum 
Table 2 Statistics and the upper limit of the maximum value of the response velocity spectrum

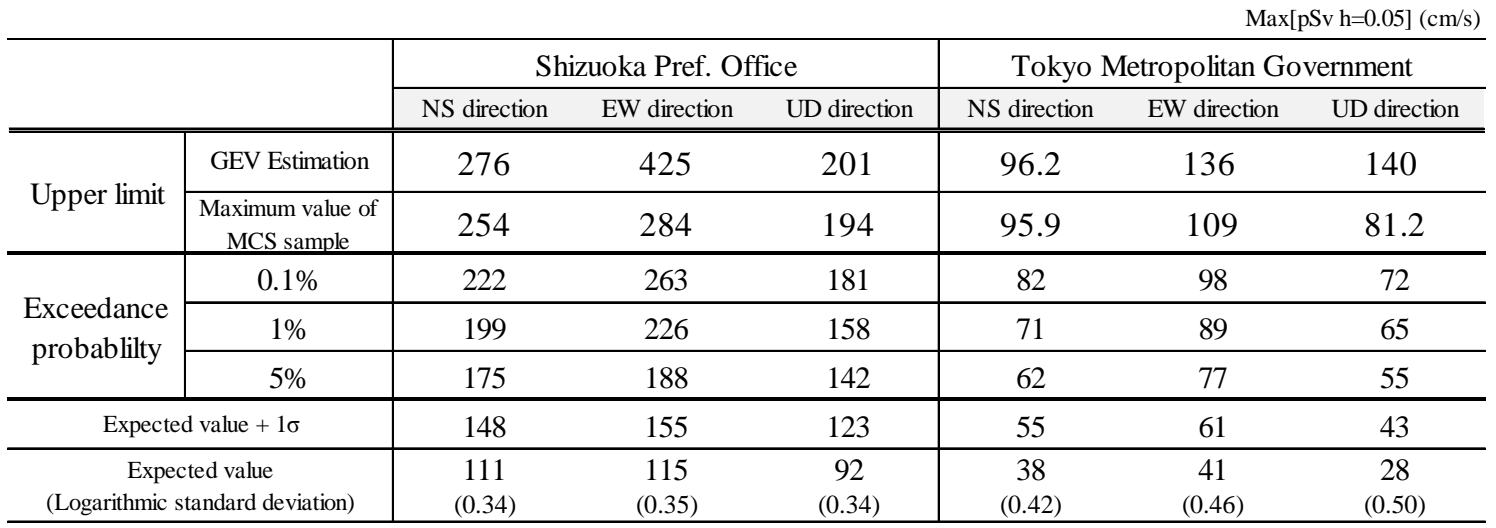

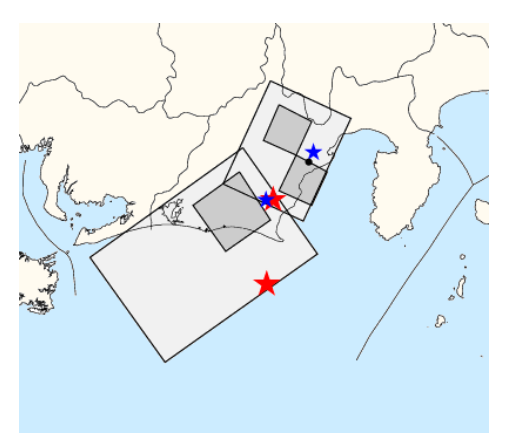

(a) NS direction comp.

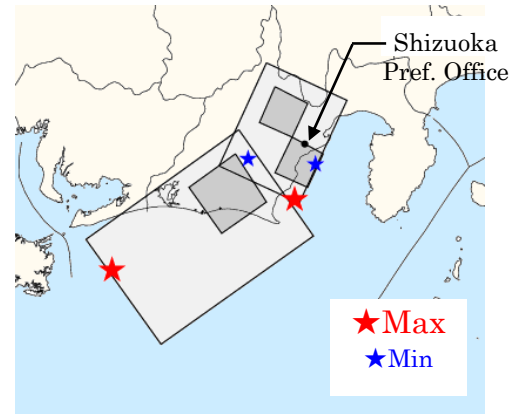

(b) EW direction comp.

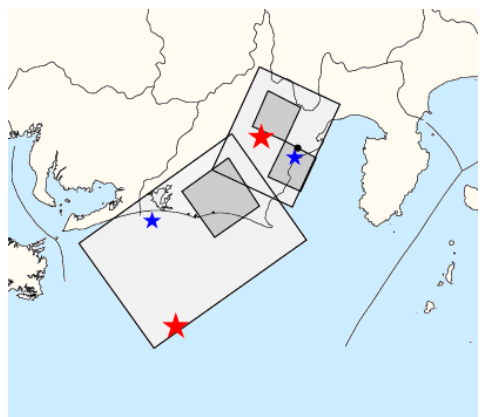

(c) UD direction comp.

Fig. 6 Rupture starting point which gives max/min of the velocity response spectrum of Shizuoka Pref. Office

\section{Examination of the simple model using closed-form solution}

With regard to the aforementioned MCS, Fig. 6 shows the rupture starting point for each direction component, where the maximum velocity response spectrum value on the calculation point at Shizuoka Pref. Office is calculated as the value of maximum or minimum in the samples. It seems the long-period ground motion tends to increase when the rupture starting point, the asperity, and the observation point are aligned in a straight line in a plane.

Even though it is very difficult to obtain a closed-form explicit analytical solution of the probability density of the velocity response spectrum value or the maximum value in the long period region, here we consider a rough and approximate indicator, $\Theta$, in a square focal region with the central asperity as shown in Fig. 7. $\Theta(0<\Theta<180$ degrees $)$ is the angle between the line connecting the rupture starting point and the asperity center, and the line connecting the asperity center and the calculation point. If $\Theta$ is less than 90 degrees, i.e., when viewed from the rupture starting point where the calculation point is on the opposite side of the asperity, then it is estimated that the ground motion is not so strong, but it becomes stronger as $\Theta$ approaches 180 degrees. Therefore, it is expected that the probability density function of the ground motion is similar to the probability density of $\Theta$, and $\Theta$ may serve as a reference indicator of the long-period ground motion intensity. If the rupture starting point is uniformly distributed in the lower half of the square fault and the asperity is located in the center of the fault, the cumulative distribution and the probability density function of $\Theta$ are expressed as follows. 


$$
\begin{gathered}
F_{\Theta}(\theta)=P[\Theta \leq \theta]=P\left[\frac{l \times l \tan \theta}{2} \leq 2 l^{2}\right]=\frac{\tan \theta}{4} \\
f_{\Theta}(\theta)=\frac{d F_{\Theta}(\theta)}{d \theta}=\frac{1}{4 \cos ^{2} \theta}
\end{gathered}
$$

The reason for the assumption that the rupture starting point is distributed in the lower half of the fault plane is that the actual starting point in rupture (as mentioned in reference 1) tends to take such a situation. In addition, it is clear that the ground motion does not become so strong from the tripartite positional relationship between the rupture starting point, the asperity, and the calculation point when the rupture starting point is in the upper half of the fault $(\Theta<90$ degrees). As shown in Fig. 7, the probability density of $\Theta$ has the peak at $\theta=135$ degrees, i.e. the rupture starting point is on the diagonal of the rectangle as can be understood geometrically, and $\Theta$ has the same probability density around $\theta=90$ degrees and $\theta=180$ degrees. Naturally, there exist the minimum and maximum values, $\theta=90$ and $\theta=180$ degrees, respectively. However, the probability density of $\Theta$ is completely uniform when considering a fault plane of a circular shape as shown in Fig. 8. It follows that the probability density of the ground motion is deeply dependent on the spatial distribution characteristics of the rupture starting point. It should therefore be noted that the first approximation assumption has a strong influence on the results as in this study in which the rupture starting point was assumed to be uniformly distributed in the rectangular shape fault plane. In addition, in the probability density plots shown in Figs. 7 to 9, the solid lines express the above-mentioned analysis solutions, and the symbols express the numerical solutions by MCS with a sample size of 100,000.

Referring to Fig. 6, the distance to the asperity center from the rupture starting point, R, is related to the intensity of the ground motion, and the calculated long-period ground motion appears to be strong when $\mathrm{R}$ is long. In the case of a square fault plane, the probability density of $\mathrm{R}$ is given by

$$
\begin{aligned}
f_{R}(r) & =\frac{2 \pi}{L^{2}} r \quad(0 \leq \mathrm{r}<\mathrm{L} / 2) \\
& =\left(\frac{\pi}{4}-\cos ^{-1} \frac{L}{2 r}\right) \times \frac{8 r}{L^{2}} \quad(\mathrm{~L} / 2 \leq \mathrm{r} \leq \mathrm{L} / \sqrt{2})
\end{aligned}
$$

Figure 9 shows $f_{R}(r)$. If the extreme value of the ground motion depends on $\mathrm{R}$, the tails of the distribution are expressed in the form of the lower part of Eq. (8). If $\Theta$ is dominant in the intensity of the ground motion, the probability density should drop step-like on the upper side as in Fig. 7, but the MCS results did not, as described in the previous section. Therefore, $\mathrm{R}$ also appears to have a certain effect. This suggests that the ground motion at the calculation point can become stronger when the rupture starting point is set at B-point rather than at A-point in the virtual fault shown in Fig. 10. It seems that this is related to the effect of the rupture propagation mode (the mode of spread of the rupture front within the asperity) on the ground motion at the calculation point.

\section{Examination of a simple model using numerical solution}

In this section, the statistic distribution of long-period ground motion is calculated by the numerical analysis of a simple source model. In the analysis, the following cases are examined: Case A in which the rupture starting point is uniformly distributed over the whole fault plane, Case B in which the rupture starting point is uniformly distributed over the lower half of the fault, and Case $\mathrm{C}$ in which the rupture starting point is uniformly distributed over the lower half of the fault except the asperity area. The specifications of the model are shown in Fig. 11. The sample size of the MCS is 10,000. The slip velocity function and the stress drop of the asperity and background area were the same as those for the Tokai earthquake model of this study, but here we focus only on the shape of the statistical distribution of the long-period ground motion with uncertainty of the rupture starting point. Therefore, the result values of the velocity response spectra have no meaning. 

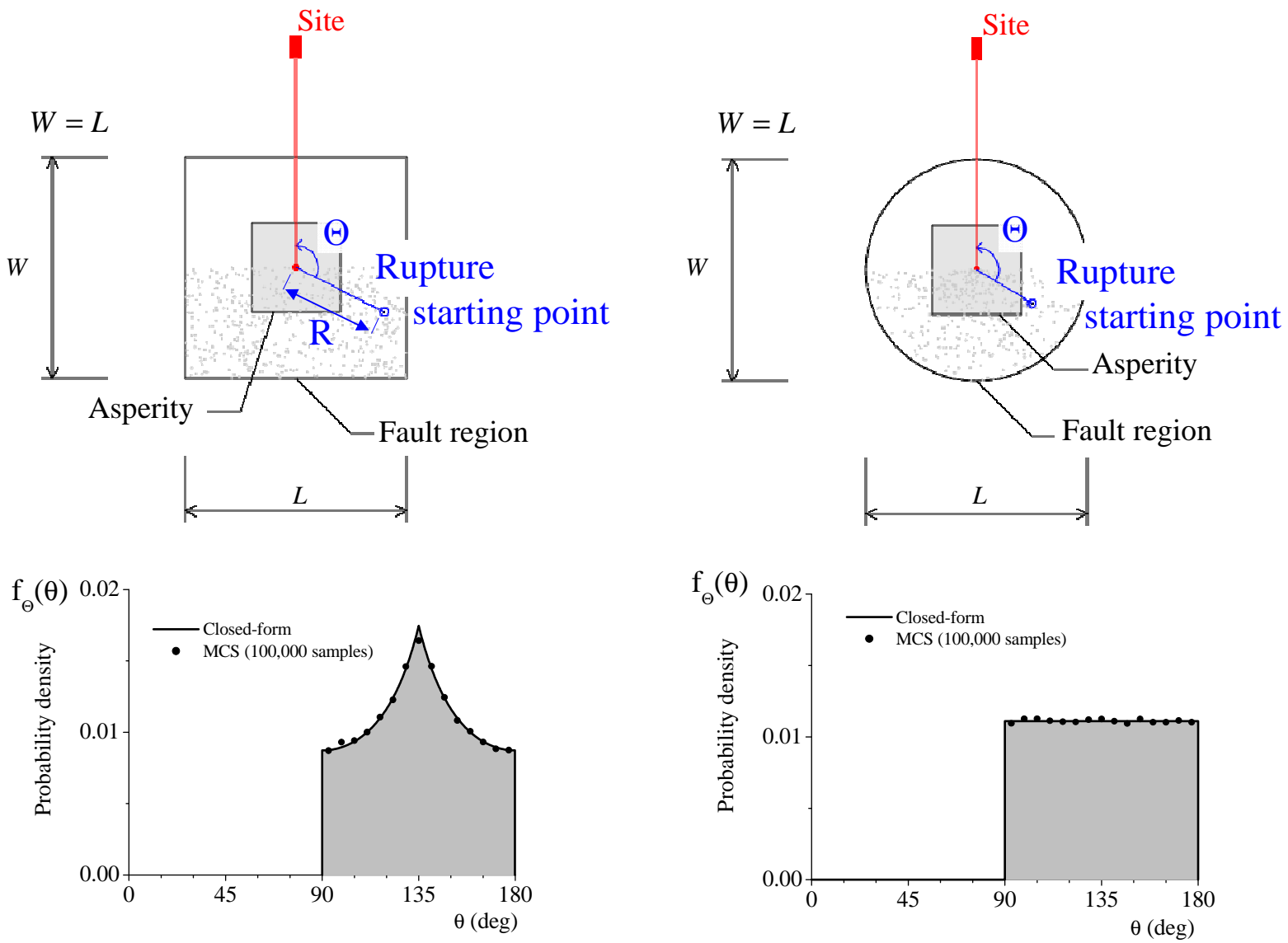

Fig. 7 Probability density of $\Theta$

(Square fault region)

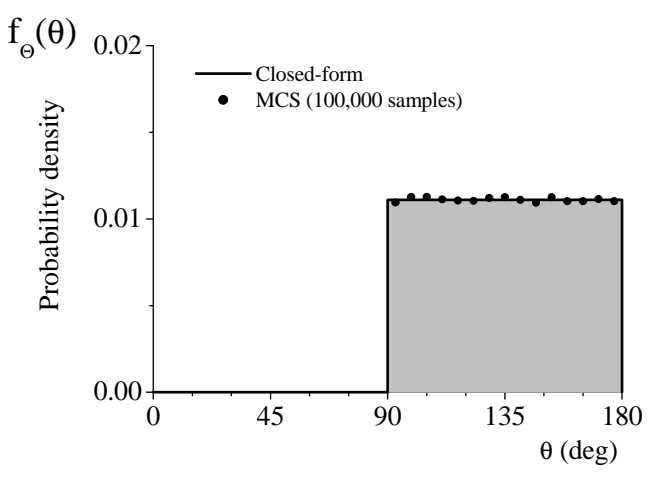

Fig. 8 Probability density of $\Theta$ (Circular fault region)

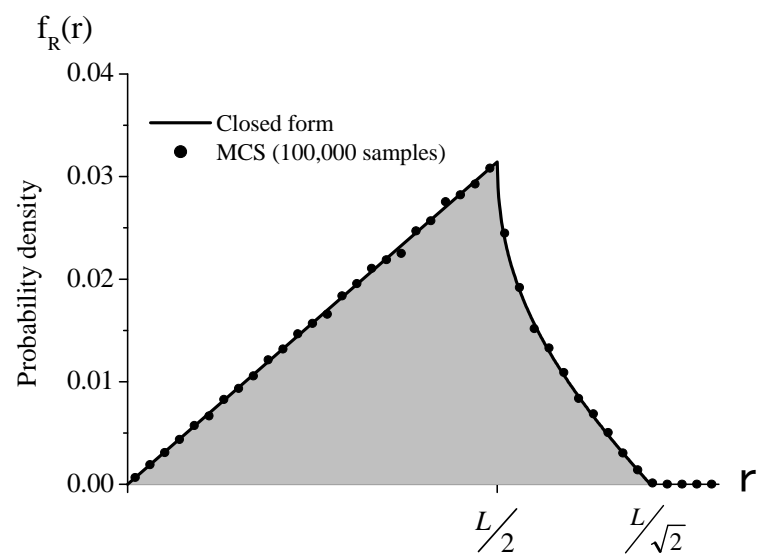

Fig. 9 Probability density of $\mathrm{R}$ (Square fault region)

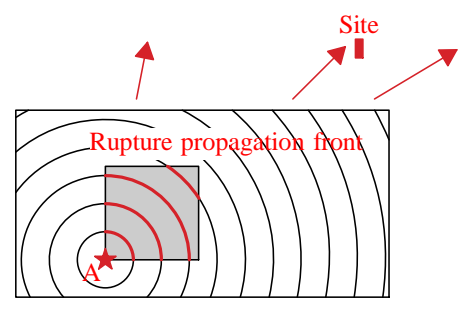

(a) Lower bound of asperity

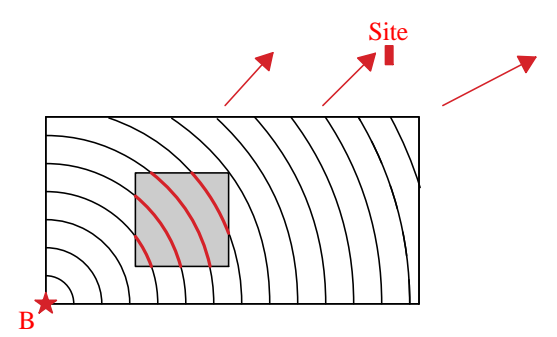

(b) Lower bound of fault region

Fig. 10 Rupture starting point of virtual fault 


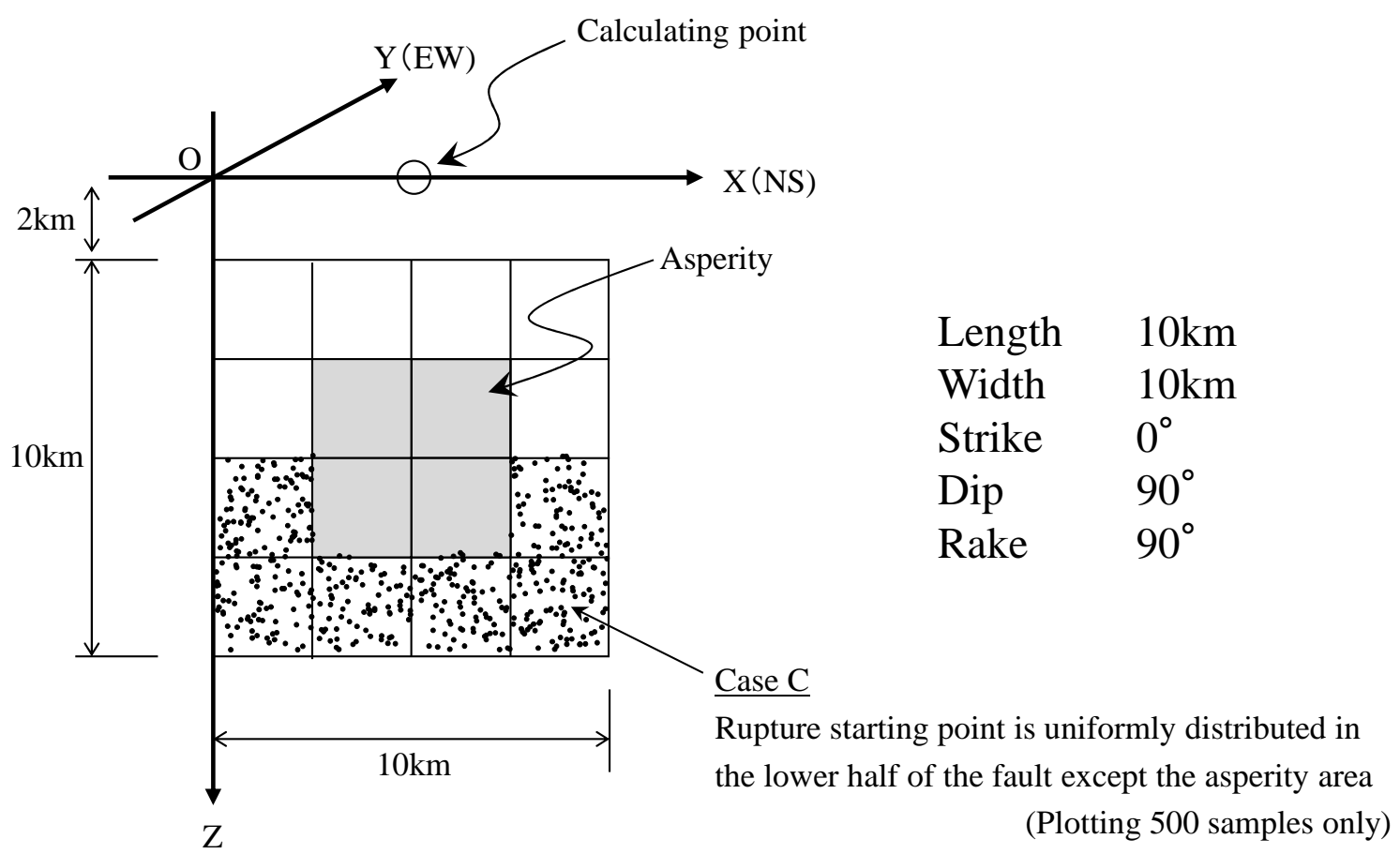

Fig. 11 Simple model for numerical solution

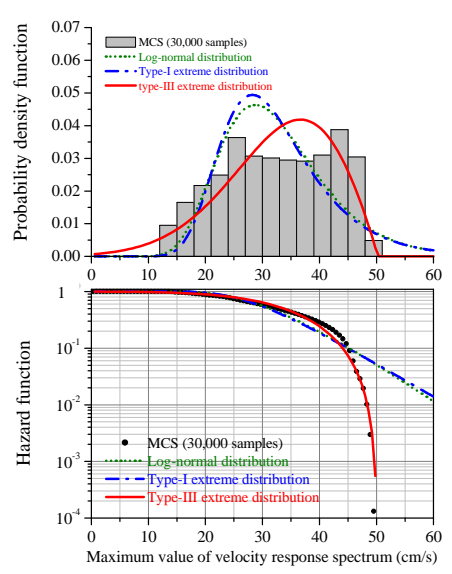

(a) NS direction Comp.

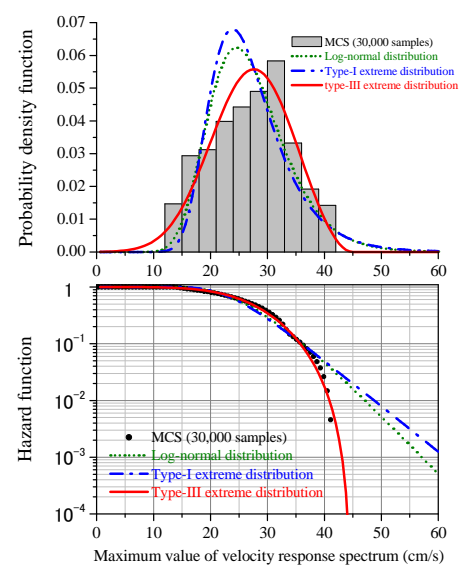

(b) EW direction Comp.

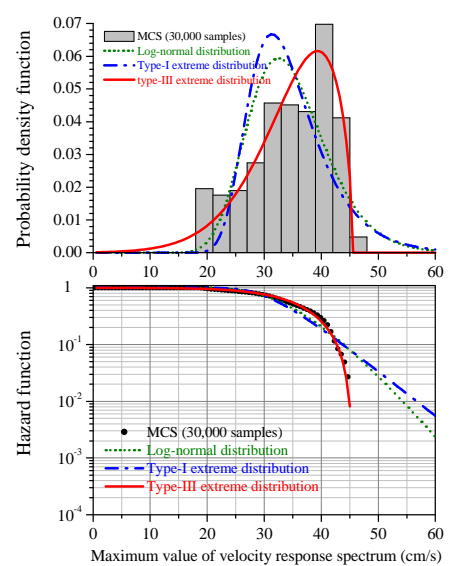

(c) UD direction Comp.

Fig. 12 Case A: Probability density and hazard function of the maximum value of velocity response spectrum
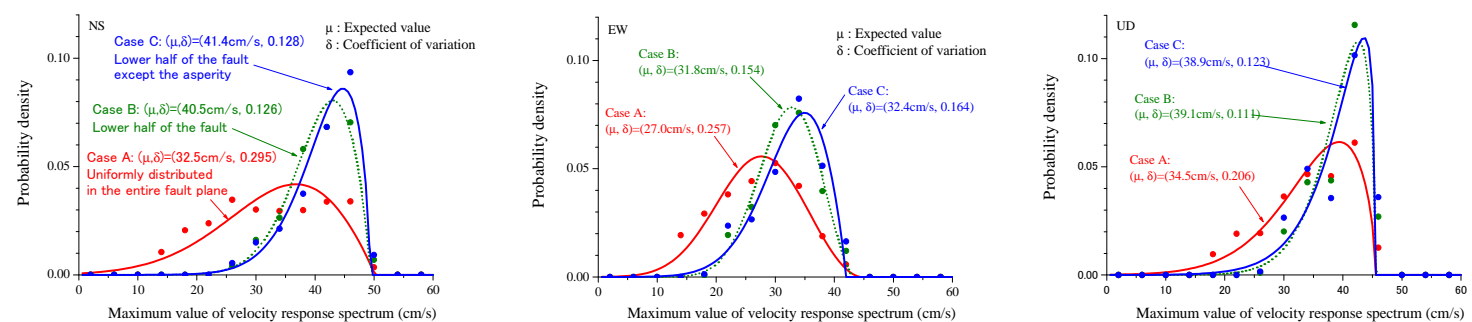

Fig. 13 Comparison of probability density function (Case A to Case C) 
Figure 12 shows the statistic distribution of the maximum values of the velocity response spectra for the 3.5 to $20 \mathrm{sec}$ period region. Similarly to the above-mentioned simulation, the MCS results using the simple model are well represented by TYPE-III extreme value distribution. Figure 13 shows the MCS results of Cases A to C (symbols), and the estimation by TYPE-III extreme value distribution (solid lines). Case B, which assumed that the rupture starting point is distributed over the lower half area of the fault, includes no $\Theta<90$ case, which leads to weak ground motion. The distribution of the maximum response values is therefore skewed toward the large side, and the coefficient of variation is found to be smaller accordingly. Furthermore, the distribution of Case $\mathrm{C}$ that does not assume the rupture start point upon the asperity tends to be biased further toward larger values.

From the result of the analysis in this section, it can safely be said that the rupture starting point may be assumed to be in the lower part of a fault, when discussing the variability of calculated ground motion due to uncertainty in the location of the rupture starting point, as far as the maximum values of the velocity response spectra of long-period ground motion are concerned. It may also be no problem to assume the rupture starting point to be in the limited area of the fault excepting the internal part of the asperity. From a different perspective, it is unnatural to assume that rupture begins in the upper part of the fault and its effect propagates down to the lower part, as the soil stiffness is generally lower at higher levels and higher at lower levels, particularly when considering a fault with a large dip angle as dealt with in this study. A limited area of the rupture starting point and reduced number of computation cases achieved by this technique are significant for seeking its application to seismic design practice.

\section{CONCLUSIONS}

Using the expected Tokai Earthquake as an example, the authors numerically examined the effect of uncertainty (variation) related to the location of the rupture starting point on the calculated long-period ground motion by a source model. Although the calculation results of this study based on the wavenumber integration method assuming a layered soil model did not agree precisely with the results published by the Headquarters for Earthquake Research Promotion using a 3D soil structure model, this simple model was found to provide prediction values of the maximum velocity response spectra with a certain degree of accuracy at a low calculation cost. Monte Carlo simulation with a sample size of 30,000 was then conducted to evaluate the statistics of long-period ground motion considering the uncertainty of the rupture starting point in the fault. This simulation showed that the statistical distribution of the maximum values of the velocity response spectra at the calculation point in the period range of 3.5 to $20 \mathrm{sec}$ was well expressed by TYPE III extreme value distribution. The presence of an upper limit in this statistical distribution was also pointed out by using the extreme value statistics theory.

It is inevitable that uncertainty is involved in fault parameters, bringing a lot of difficulty, even with all our knowledge of earthquakes, in evaluating the tails of the probabilistic distribution of ground motion, which must be used for seismic engineering. Computed ground motion is also strongly affected by the assumption of the slip velocity function, which was not dealt with in this study. Many other parameters contribute to the intensity of long-period ground motion, such as the fault depth, dip angle, and slip value of the asperity. Therefore, the exceedance probability values related to a single parameter presented here may have no absolute meaning. Nevertheless, it is not at all a waste of time to think stochastically. It is therefore considered useful for future progress of seismic design to stochastically investigate the effects of various parameters on strong ground motion, while accumulating a lot of seismological knowledge.

\section{ACKNOWLEDGMENT}

The authors would like to thank Dr. Hiroe MIYAKE, Earthquake Research Institute, the University of Tokyo. She gave us valuable advice and materials for setting the model of the Tokai Earthquake source. 
This study was conducted by the joint research titled "The strong ground motion prediction of the next great earthquake" sponsored by seven companies (Hazama Ando Corporation, Kumagai Gumi Co., Ltd., Sato Kogyo Co., Ltd., Fujita Corporation, and Maeda Corporation). They would also like to thank the working group members for their active and useful discussion, as well as the research corporation, and the parties concerned of each company for their support for this program.

\section{REFERENCES}

1) Midorikawa, S., H. Miura, and T. Itoi (2008). "Variability of ground motion intensity by stochastic Green's function method, Part I Statistical characteristics of fault parameters." Summaries of technical papers of annual meeting Architectural Institute of Japan, 2008, 867-868.

2) Sekiguchi, H., M. Yoshimi, K. Yoshida, and H. Horikawa (2006). "Multi-scale heterogeneous model for broad-band ground motion prediction of great earthquake in subduction zone."

Summaries of technical papers for joint symposium of Great Subduction Earthquake, Architectural Institute of Japan and Japan Society of Civil Engineers, 2006.2, 67-79.

3) Okano, H. (2011). "Dispersion of the predicted earthquake and the simulated earthquake wave for structural design." Summaries of technical papers for symposium of Design seismic load considering active fault, Architectural Institute of Japan, 2011.8, 27-40.

4) Earthquake Research Committee, The Headquarters for Earthquake Research Promotion (2009). "Long Period Ground Motion Hazard Maps of Japan (2009 trial version)." http://www.jishin.go.jp/main/chousa/09_choshuki/index.htm

5) Miyake, H., K. Koketsu, and T. Furumura (2008): Source modeling of subduction-zone earthquakes and long-period ground motion validation in the Tokyo metropolitan area, Proceedings of the 14th World Conference on Earthquake Engineering, Paper No. S10-012.

6) Koketsu, K., H. Miyake, H. Fujiwara, and T. Hashimoto (2008): Progress towards a Japan integrated velocity structure model and long-period ground motion hazard map, Proceedings of the 14th World Conference on Earthquake Engineering, Paper No. S10-038.

7) Yamada, M., S. Senna, and H. Fujiwara (2007). "Statistical analysis of predicted ground motions on the basis of a recipe for strong-motion prediction. For variety of source parameters " Journal of Japan Association for Earthquake Engineering Vol. 7 No. 1, 43-60.

8) Nakamura, H., and T. Miyatake (2000). "An approximate expression of slip velocity time function for simulation of near-field strong ground motion" Zishin 53, 1-9.

9) Hisada, Y. (2000). "Efficient methods for computing Green's function and normal mode solutions for layered half spaces." Journal of Structural and Construction Engineering, Architectural Institute of Japan, Vol. 501, 49-56.

10) Furumura, T. (2008). "The long period motions to grow in the Kanto Plain." News letters, Japan Geoscience Union, Vol. 4, No. 2, 5-7.

11) Earthquake Research Committee, The Headquarters for Earthquake Research Promotion (2009). "National seismic hazard maps for Japan (2009.7.21 release)" Technical report appendix 3, Strong ground motion prediction method of earthquake identified the source fault ("recipe") http://www.jishin.go.jp/main/chousa/09_yosokuchizu/index.htm

12) Japan Society of Civil Engineers. "Special task committee of earthquake resistance of civil engineering structures" Second proposal, Commentary, Chapter 2, Earthquake and earthquake motions that need to be considered in earthquake-resistant design. http://www.jsce.or.jp/committee/earth/chap2.html

13) Takemura, M. (2006). "Prediction method for strong ground motion and earthquake wave for seismic design: Perspective and Task" Bulletin of Japan Association for Earthquake Engineering, No. 4, 2006.7, 26-30.

14) Somerville, P. G., K. Irikura, R. Graves, S. Sawada, D. Wald, N. Abrahamson, Y. Iwasaki, T. Kagawa, N. Smith, and A. Kowada (1999). "Characterizing crustal earthquake slip models for the 
prediction of strong ground motion", Seismological Research Letters, Vol. 70, 59-80.

15) Kikuchi, M. (2003). "Real time seismology. Chapter 4.3 Microstructure of the source" University of Tokyo Press, 2003, 126-131

16) Central Disaster Management Council: Committee on Tonankai, Nankai Earthquake (2006). "About the distribution of inland earthquake seismic intensity of Chubu, Kinki area. Chapter 2 Seismic source modeling", 7 Dec., 2006

http://www.bousai.go.jp/kaigirep/chuobou/senmon/tounankai_nankaijishin/26/pdf/siryou1.pdf

17) Ito, M., H. Kameda, and K. Kuroda Co-translation (1988), Alfredo H-S. Ang, Wilson H. Tang:

"Probability Concepts in Engineering planning and design", Maruzen Co., Ltd., 1988, 207-256

18) Minotani, C. (2010), "Statistical Distribution Handbook", Asakura Publishing Co., Ltd., 292-295

(Original Japanese Paper Published: February, 2014)

(English Version Submitted: April 3, 2014)

(English Version Accepted: May 1, 2014) 